\title{
Simplified Methods for Eigenvalue Assignment
}

\author{
Omar Moh'd El-Basheer El-Ghezawi \\ Electrical Engineering Department, The University of Jordan, Amman, Jordan \\ Email: ghezawi@ju.edu.jo
}

Received 20 March 2015; accepted 26 May 2015; published 29 May 2015

Copyright (C) 2015 by author and Scientific Research Publishing Inc.

This work is licensed under the Creative Commons Attribution International License (CC BY). http://creativecommons.org/licenses/by/4.0/

c) (i) Open Access

\begin{abstract}
A state feedback method of reduced order for eigenvalue assignment is developed in this paper. It offers immediate assignment of $m$ eigenvalues, with freedom to assign the remaining $n-m$ eigenvalues. The method also enjoys a systematic one-step application in the case where the system has a square submatrix. Further simplification is also possible in certain cases. The method is shown to be applicable to uncontrollable systems, offering the simplest control law when having maximum uncontrollable eigenvalues.
\end{abstract}

\section{Keywords}

Eigenvalue Assignment, Pole Placement, Recursive Methods

\section{Introduction}

The problem of eigenvalue assignment is well established in control theory where numerous methods have been proposed-each with certain advantages and disadvantages. However, a need still arises for methods which are simple in concept and can be easily implemented. A fulfillment to such need is contributed by this paper.

As compared with some previous methods for eigenvalue assignment, this method doesn't require specific transformations, knowledge of the open loop eigenvalues or the determination of the closed loop eigenvectors. The method utilizes submatrices stemming from a particular state transformation. The transformation is only needed in the development of the method and not the actual assignment of the eigenvalues.

The proposed method tackles eigenvalue assignment by manipulating lower order matrices, hence enjoying some numerical advantages. Furthermore, $m$ eigenvalues are assigned independently of the remaining $n-m$ eigenvalues. The method is simplified when $n=2 m$, where $m$ is the rank of $B$, resulting in a systematic feedback law requiring only the specification of two $m x m$ matrices. It can be further simplified in cases where the columns of $B$ and $A B$ constitute an invertible matrix. 
The method is also shown to apply to uncontrollable systems where certain features of some submatrices are pointed out, thus providing additional degrees of freedom in the control law. Furthermore, in the case of maximum number of uncontrollable eigenvalues, the controller is shown to exhibit its simplest form and offer arbitrariness which may be utilized in fulfilling a myriad of design objectives.

Finally, the systematic and straightforward nature of the method is demonstrated by two examples.

\section{The Nonrecursive Feedback Law}

The assignment law considered is a state feedback law of the form $u=-K x$ applied to the system

$$
\dot{x}=A x+B u
$$

where $x \in \mathbb{R}^{n}, u \in \mathbb{R}^{m}$, the rank of $B$ is $m, R($.$) and null(.) refer to the range and null spaces of (.).$

For the development of the simplified methods, a state transformation $T$ is used where $x=T z$, leading to system and input matrices of the form conformal with those in [1].

$$
\dot{z}=F z+B_{z} u
$$

where

$$
F=T^{-1} A T=\left[\begin{array}{ll}
F_{1} & F_{2} \\
F_{3} & F_{4}
\end{array}\right] \text { such that } B_{z}=T^{-1} B=\left[\begin{array}{c}
I_{m} \\
0_{m x m}
\end{array}\right]
$$

Such requirement on $T^{-1} B$ necessitates $T=\left[\begin{array}{ll}B & N\end{array}\right]$ where $N$ is an $n x n-m$ matrix chosen to ensure the nonsingularity of $T$. The inverse of $T$ is represented by

$$
T^{-1}=\left[\begin{array}{c}
B^{g} \\
N^{g}
\end{array}\right]
$$

where $B^{g}$, and $N^{g}$ can be looked upon as a name for that partition of $T^{-1}$ related to $B$ and $N$ or as unique generalized inverses of matrices [2] [3]. The generalized inverses are unique in our case since they satisfy the additional conditions

$$
B^{g} B=I_{r}, \quad N^{g} N=I_{n-r}, B^{g} N=0 \text {, and } N^{g} B=0
$$

Using the terminology above, the submatrices become

$$
F_{1}=B^{g} A B, F_{2}=B^{g} A N, F_{3}=N^{g} A B \text {, and } F_{4}=N^{g} A N
$$

In addition

$$
B B^{g}+N N^{g}=I_{n}
$$

With reference to the recursive method of Hassan et al. [1], $m$ eigenvalues are assigned through an $m x m$ matrix $\Lambda_{m}$ while the remaining $n-m$ eigenvalues are assigned through the reduced order matrix pair $\left(F_{4}, F_{3}\right)$ i.e. the $n-m$ eigenvalues to be assigned are eigenvalues of $F_{4}-F_{3} K^{*}$. The reduced order matrix $K^{*}$ is determined independently of $\Lambda_{m}$.

The recursive method [1] is now manipulated to result in a non-recursive feedback law.

According to [1]; having undergone all recursive steps the final feedback matrix is given by

$$
K=\left(D F-\Lambda_{m} D\right) T^{-1}
$$

where

$$
D=\left[\begin{array}{ll}
I_{m} & K
\end{array}\right]
$$

i.e.

$$
K=\left(\left[\begin{array}{ll}
I_{m} & K^{*}
\end{array}\right] F-\Lambda_{m}\left[\begin{array}{ll}
I_{m} & K^{*}
\end{array}\right]\right) T^{-1}
$$

substituting $F$ as given in (2.3) and $T^{-1}$ as given in (2.4) yields

$$
K=\left[F_{1}+K^{*} F_{3}-\Lambda_{m} \quad F_{2}+K^{*} F_{4}-\Lambda_{m} K^{*}\right] T^{-1}
$$




$$
K=\left[F_{1}+K^{*} F_{3}-\Lambda_{m}\right] B^{g}+\left[F_{2}+K^{*} F_{4}-\Lambda_{m} K^{*}\right] N^{g}
$$

Substituting the values of $F_{1}, F_{2}, F_{3}$, and $F_{4}$ as in (2.6), gives

$$
\begin{gathered}
K=B^{g} A B B^{g}+K^{*} N^{g} A B B^{g}-\Lambda_{m} B^{g}+B^{g} A N N^{g}+K^{*} N^{g} A N N^{g}-\Lambda_{m} K^{*} N^{g} \\
K=B^{g} A\left(B B^{g}+? N N^{g}\right)+K^{*} N^{g} A\left(B B^{g}+N N^{g}\right)-\Lambda_{m}\left(B^{g}+K^{*} N^{g}\right)
\end{gathered}
$$

Using the fact that $B B^{g}+N N^{g}=I_{n}$ as in (2.7), the equation can be finally put in the form

$$
K=\left(B^{g}+K^{*} N^{g}\right) A-\Lambda_{m}\left(B^{g}+K^{*} N^{g}\right)
$$

The advantage of this feedback law as given in (2.10) is that assignment of $n$ eigenvalues is split into independent assignment of $m$ eigenvalues through $\Lambda_{m}$ and assignment of $n-m$ eigenvalues of $F_{4}-F_{3} K^{*}$ through a suitable $K^{*}$. Existing non-recursive methods not requiring state transformation like [4] and [5] or any other eigenvalue assignment method can be used to determine $K^{*}$. In addition, since $F_{4}$ has dimension $n-m$, the matrix $K^{*}$ has a reduced dimension $m x n-m$. Further utilization of (2.10) is to be followed in Section 6 when it comes to assignment of uncontrollable eigenvalues, where it is shown that a further reduction in the order of $K^{*}$ is possible to the extent that $K^{*}$ can be taken as zero in certain cases.

\section{A Simplified Method When $n=2 m$ and the System Is Controllable}

Although the previous development resulted in a controller which manipulates lower order matrices; the selection of $K^{*}$ remains an eigenvalue problem to be solved. Known methods of eigenvalue assignment can be used with the benefit of dealing with reduced order matrices, see [6]. However, further simplification can be made in the case where $n=2 \mathrm{~m}$ and $F_{3}$ is invertible as developed below.

Due to the presence of identical terms within the parenthesis's, we simplify one term in the state feedback matrix

$$
K=\left(B^{g}+K^{*} N^{g}\right) A-\Lambda_{m}\left(B^{g}+K^{*} N^{g}\right)
$$

where $\Lambda_{m}$ assigns $m$ eigenvalues and $K^{*}$ assigns the remaining $n-m$ eigenvalues through

$$
F_{4}-F_{3} K^{*}=\Lambda_{n-m}
$$

Assuming the nonsingularity of $F_{3}$ and that the remaining $n-m$ eigenvalues are eigenvalues of the matrix $\Lambda_{n-m}$, then

$$
K^{*}=F_{3}^{-1}\left(F_{4}-\Lambda_{n-m}\right)
$$

Substituting the value of $K^{*}$ in any term within a parenthesis of (2.10) gives

$$
\begin{aligned}
B^{g}+F_{3}^{-1}\left(F_{4}-\Lambda_{n-m}\right) N^{g} & =B^{g}+F_{3}^{-1} F_{4} N^{g}-F_{3}^{-1} \Lambda_{n-m} N^{g} \\
& =B^{g}+F_{3}^{-1} N^{g} A N N^{g}-F_{3}^{-1} \Lambda_{n-m} N^{g}
\end{aligned}
$$

Using (2.7), and recalling $F_{3}=N^{g} A B$

$$
\begin{aligned}
& =B^{g}+F_{3}^{-1} N^{g} A-F_{3}^{-1} N^{g} A B B^{g}-F_{3}^{-1} \Lambda_{n-m} N^{g} \\
& =F_{3}^{-1} N^{g} A-F_{3}^{-1} \Lambda_{n-m} N^{g} \\
& =F_{3}^{-1}\left(N^{g} A-\Lambda_{n-m} N^{g}\right)
\end{aligned}
$$

substituting this value for the two terms in the parenthesis's in Equation (2.10) gives

$$
K=F_{3}^{-1}\left(N^{g} A-\Lambda_{n-m} N^{g}\right) A-\Lambda_{m} F_{3}^{-1}\left(N^{g} A-\Lambda_{n-m} N^{g}\right)
$$

Some remarks regarding the control law are stated below.

- A necessary condition for the invertibility of $F_{3}$ is the controllability of the system.

To see this, suppose $F_{3}$ is nonsingular and the system is uncontrollable, then according to (3.4) it is possible to change all $n$ eigenvalues of $A$, contradicting the established fact that uncontrollable eigenvalues cannot be 
changed by state feedback. Hence, only if the system is controllable will $F_{3}$ be nonsingular.

- No need to do the state transformation. The determination of (2.4) is only needed to extract $N^{g}$ and to subsequently evaluate the inverse of $F_{3}$ (equals $N^{g} A B$ ).

- Assignment of $n$ eigenvalues is achieved through $\frac{n}{2} \times \frac{n}{2}$ lower order $\Lambda_{m}$ and $\Lambda_{n-m}$ matrices, which can be diagonal, Jordan forms, or skew-symmetric when it comes assignment of complex eigenvalues.

- As compared with other assignments laws the highest power of $A$ involved is two while it's $n$ for certain celebrated methods like Ackermann's method [7]. This gives numerical advantages in terms of reducing matrix multiplication rounding errors, as demonstrated by Petkov [8], who showed that matrix multiplications is ill conditioned.

\section{Further Simplification}

Additional simplification can be done to the form of (3.4). By replacing $\Lambda_{m}$ by $\mathrm{F}_{3}^{-1} \Lambda_{M} F_{3}$ where $\Lambda_{m}, \Lambda_{M}$, and $\mathrm{F}_{3}^{-1} \Lambda_{M} F_{3}$ have the same set of eigenvalues,

$$
\begin{gathered}
K=F_{3}^{-1}\left(N^{g} A-\Lambda_{n-m} N^{g}\right) A-F_{3}^{-1} \Lambda_{M} F_{3} F_{3}^{-1}\left(N^{g} A-\Lambda_{n-m} N^{g}\right) \\
K=F_{3}^{-1}\left(N^{g} A^{2}-\Lambda_{n-m} N^{g} A-\Lambda_{M} N^{g} A+\Lambda_{M} \Lambda_{n-m} N^{g}\right)
\end{gathered}
$$

Ending up with a compact form for $K$ as

$$
K=F_{3}^{-1}\left(N^{g} A^{2}-\left(\Lambda_{M}+\Lambda_{n-m}\right) N^{g} A+\Lambda_{M} \Lambda_{n-m} N^{g}\right)
$$

- If $N$ is chosen as a matrix representation of $\operatorname{null}\left(B^{T}\right)$, then $N^{g}$ can be obtained independently of $B$, see Lancaster [9] and Schott [10]. Also, theorem 6.4.5 pp 115 of Graybill's book [2] states, if $T=\left[\begin{array}{ll}B & N\end{array}\right]$ and $B^{T} N=0$, then $B^{g}$ and $N^{g}$ can be determined independently as $B^{g}=\left(B^{T} B\right)^{-1} B^{T}$ and

$N^{g}=\left(N^{T} N\right)^{-1} N^{T}$ respectively. The left inverse of $N^{g}$ now involves an inverse of an $n-m x n-m$

symmetric $N^{T} N$ matrix instead of the inverse of the generally non-symmetric nxn $T$ matrix needed to extract $N^{g}$.

- The choice of $N=$ matrix representation of null $\left(B^{T}\right)$ has many advantages.

- The selection of $N$ is systematic.

- Such choice gives the advantage of inverting an $n x n$ matrix through inversion of symmetric $\frac{n}{2} \times \frac{n}{2}$ matrices; thus providing numerical advantages.

- Further computational advantages are gained if the Gram-Schmidt ortho-normalization procedure is used (can be easily programmed on a digital computer and is already within the MATLAB function library). In this case, if $N=$ matrix representation of $\operatorname{null}\left(B^{T}\right)$ is orthonormal, then $N^{g}=N^{T}$.

A further simplification to (4.1) is possible in the case where $N$ is taken as $A B$. In which case, $F_{3}$ becomes the unity matrix offering a more simplified form given by.

$$
K=(A B)^{g} A^{2}-\left(\Lambda_{M}+\Lambda_{n-m}\right)(A B)^{g} A+\Lambda_{M} \Lambda_{n-m}(A B)^{g}
$$

So, the design process now reduces to the selection of $\Lambda_{m}$ and $\Lambda_{n-m}$ which specify the desired eigenvalues and the calculation of $(A B)^{g}$ according to (2.5).

\section{The Uncontrollable Case}

The non-recursive feedback law can still be applied when the system is uncontrollable. In our case, and as has been shown by [11], the pair $F_{4}$ and $F_{3}$ is the uncontrollable pair, i.e. the uncontrollable eigenvalues are eigenvalues of $F_{4}$. 
For the case $n=2 m$, the uncontrollability of the system implies the following:

a) The matrix $F_{3}$ has to be a singular matrix, otherwise an $F_{3}$ exists which can reassign arbitrarily all eigenvalues of $F_{4}$. This, together with the $m$ arbitrary eigenvalues assigned by $\Lambda_{m}$ makes the total number of arbitrarily assigned eigenvalues $n$, an impossibility for an uncontrollable system as proved in the control literature.

b) Since $F_{3}$ has to be singular, then it has columns which are scalar multiple of each other, or linear combinations of each other. To see this, due to uncontrollability, the matrix $\left[\begin{array}{ll}B & A B\end{array}\right]$ is an $n x n$ square matrix which can never have the full rank $n$. Since $B$ has necessarily rank $m=n / 2$, this leaves $A B$ with a rank less than $m$, indicating a dependence of $R(A B)$ on $\mathrm{R}(B)$ and since $F_{3}=N^{g} A B$ and $N^{g} B=0$ then $N^{g}$ may annihilate $A B$. In the case of annihilation, $F_{3}$ will have at least a zero column, say the $q$ th column. Such fact renders the qth row of $K^{*}$ immaterial since the product $F_{3} K^{*}$ will not depend on that row. This provides arbitrariness in the qth row of $K^{*}$ which can be utilized further in the design of the controller. It can lead to manipulating lower order matrices within $K^{*}$, gaining calculation efficiency.

In the light of the above facts since a nonsingular $F_{3}$ doesn't exists, the formula given in (3.4) cannot be used. Instead, any eigenvalue assignment method available in the control literature (see [12]-[14]) can be used to calculate $K^{*}$ with the advantage of dealing with matrices of reduced order.

\section{Justification of $K^{*}=0$ for the Case of Maximum Number of Uncontrollable Eigenvalues}

If the system has the maximum number of $n-m$ uncontrollable eigenvalues, then $F_{3}$ is identically the zero matrix. This has to be the case, otherwise, a nonzero $F_{3}$ is capable of changing some of these eigenvalues, an impossibility since the total number of uncontrollable is assumed to be $n-m$.

However, although (3.4) cannot be used to get the final feedback matrix $K$, a most simple form of (2.10) is now considered. The simplicity hinges on letting $K^{*}=0$. That is.

$$
K=B^{g} A-\Lambda_{m} B^{g}
$$

The justification for this form stems from the fact that in our case all uncontrollable eigenvalues are those of $F_{4}$, and can be specified by $\Lambda_{n-m}$ which can be $F_{4}$ itself, in which case, and according to (3.1), $F_{3} K^{*}$ will be zero, in which case $K^{*}$ can be taken as zero. Substituting $K^{*}=0$ in (2.10) results in (6.1).

Seeing it differently, since in our case $F_{3}$ is identically zero, this makes the product of $F_{3} K^{*}$ zero. This renders the value of $K^{*}$ immaterial, so any $K^{*}$ can be taken including the case $K^{*}=0$.

Note that $K$ in (6.1) doesn't depend on $N^{g}$, so we can relax the uniqueness of $B^{g}$; just requiring $B^{g} B=I_{n / 2}$. This is because there always exists a nonunique $B^{g}$ with a corresponding $N$ such that $B^{g} N=0$ as required by conditions (2.4). A systematic choice for $B^{g}$ is $B^{g}=\left(B^{T} B\right)^{-1} B^{T}$.

Note that $K^{*}$ can still be totally arbitrary. Such choice can be used to satisfy certain design requirements like controller matrix norm, sensitivity studies, eigenvector specifications, etc. For such cases, one has to resort to (2.10).

\section{Examples}

Example 1: Consider the controllable system given by

$$
A=\left[\begin{array}{cccc}
5 & 4 & 2 & -1 \\
4 & 4 & -1 & 2 \\
4 & 6 & 2 & 4 \\
1 & 0 & 3 & 1
\end{array}\right] \text { and } B=\left[\begin{array}{ll}
3 & 3 \\
0 & 2 \\
3 & 3 \\
2 & 2
\end{array}\right]
$$

It is required to assign the eigenvalues $-2,-3$ and $-5 \pm \mathrm{j} 4$.

To extract $\mathrm{F}_{3}$, MATLAB was used with $N$ taken as an orthonormal representation of $\operatorname{null}\left(B^{T}\right)$, resulting in 


$$
F=\left[\begin{array}{cccc}
0.9091 & -0.3636 & 1.0482 & -1.1472 \\
6.5 & 10.5 & -1.4487 & 1.1094 \\
4.1983 & 4.4348 & 2.514 & 2.378 \\
-2.7735 & -9.4299 & 1.312 & -1.9231
\end{array}\right]
$$

Hence, to five significant digits

$$
F_{3}=\left[\begin{array}{cc}
4.1983 & 4.4348 \\
-2.7735 & -9.4299
\end{array}\right] \text { and } F_{3}^{-1}=\left[\begin{array}{cc}
0.3455 & 0.1625 \\
0.1016 & -0.1538
\end{array}\right]
$$

The matrices $\Lambda_{m}$ and $\Lambda_{n-m}$ may be chosen as

$$
\Lambda_{m}=\left[\begin{array}{cc}
0 & 1 \\
-6 & -5
\end{array}\right] \text { and } \Lambda_{n-m}=\left[\begin{array}{cc}
-5 & 4 \\
-4 & -5
\end{array}\right]
$$

Using the control law given by (3.4) results in the following state feedback matrix

$$
K=\left[\begin{array}{cccc}
6.5185 & -0.526 & 3.9503 & 11.6531 \\
-4.4848 & 2.3398 & 6.853 & -0.193
\end{array}\right]
$$

To check, the system closed loop matrix $A_{c}=A-B K$ is

$$
A_{c}=\left[\begin{array}{cccc}
38.0097 & -1.4414 & -30.4098 & -35.3804 \\
12.9696 & -0.6797 & -14.7059 & 2.3859 \\
37.0097 & 0.5586 & -30.4098 & -30.3804 \\
23.0065 & -3.6276 & -18.6065 & -21.9203
\end{array}\right]
$$

Which has the eigenvalues $-2,-3,-5+\mathrm{j} 4$ and $-5-\mathrm{j} 4$.

Example 2: Consider the following system [15] where

$$
A=\left[\begin{array}{cccc}
2 & 3 & 2 & 1 \\
-2 & -3 & 0 & 0 \\
-2 & -2 & -4 & 0 \\
-2 & -2 & -2 & -5
\end{array}\right] ; \text { and } B=\left[\begin{array}{cc}
0 & 1 \\
1 & -2 \\
-2 & 1 \\
1 & 0
\end{array}\right]
$$

This system is uncontrollable with -1 and -4 being the uncontrollable eigenvalues. It is desired to assign the two eigenvalues -4 and -5 .

So let

$$
\Lambda_{m}=\left[\begin{array}{cc}
-4 & 0 \\
0 & -5
\end{array}\right]
$$
To expose the controllable and uncontrollable eigenvalues, we may take $N=\left[\begin{array}{llll}0 & 0 & 1 & 0 \\ 0 & 0 & 0 & 1\end{array}\right]^{\mathrm{T}}$
Yielding

$$
F=\left[\begin{array}{cccc}
-3 & 0 & 4 & 2 \\
0 & -2 & 2 & 1 \\
0 & 0 & 2 & 3 \\
0 & 0 & -6 & -7
\end{array}\right] \text {; and } B_{z}=\left[\begin{array}{cc}
1 & 0 \\
0 & 1 \\
0 & 0 \\
0 & 0
\end{array}\right]
$$

Which shows that $F_{3}=0,-2$ and -3 are the controllable eigenvalues and that the uncontrollable eigenvalues are those of $\left[\begin{array}{cc}2 & 3 \\ -6 & -7\end{array}\right]$; i.e. -1 and -4 . In fact, we need not bother finding them as they aren't needed in the calculation of $K$.

Besides, the inverse of $T$ isn't needed to extract $B^{g}$. Instead, $B^{g}$ can be taken as $B^{g}=\left(B^{T} B\right)^{-1} B^{T}$ giving 


$$
B^{g}=\left[\begin{array}{llll}
0.2 & -0.1 & -0.4 & 0.3 \\
0.3 & -0.4 & -0.1 & 0.2
\end{array}\right]
$$

Using $K$ as in (6.1) yields a state feedback $K$ matrix, say $K=K_{1}$

$$
K_{1}=\left[\begin{array}{cccc}
1.6 & 0.7 & -0.2 & -0.1 \\
2.7 & -0.1 & 0.1 & 0.3
\end{array}\right]
$$

Another $B^{g}$, just satisfying $B^{g} B=I_{m}$ with no regard to any $N$ may be

$$
B^{g}=\left[\begin{array}{llll}
0 & 0 & 0 & 1 \\
1 & 0 & 0 & 0
\end{array}\right]
$$

Which results in a different state feedback $K$ matrix, say $K=K_{2}$

$$
K_{2}=\left[\begin{array}{cccc}
-2 & -2 & -2 & -1 \\
7 & 3 & 2 & 1
\end{array}\right]
$$

Both $K_{1}$ and $K_{2}$ result in the assignment of two eigenvalues -4 and -5 and the uncontrollable eigenvalues -1 and -4 .

\section{Conclusion}

The paper has considered a method for eigenvalue assignment based on a scheme of recursive nature. The method involves algebraic manipulation of lower order matrices with an advantage of not requiring state transformation or eigenvectors determination. The method is further simplified in the case where $n=2 m$. The method is extended to deal with uncontrollable systems where it is shown that $K^{*}$ exhibits a certain degree of arbitrariness, to the extent of resulting in the simplest form for the state feedback law. The examples considered demonstrate the ease of use of the method.

\section{References}

[1] Hassan, M.M. and Amin, M.H. (1987) Recursive Eigenstructure Assignment in Linear Systems. International Journal of Control, 45, 291-310. http://dx.doi.org/10.1080/00207178708933729

[2] Graybill, F.A. (1983) Matrices with Applications in Statistics. Wadsworth Publishing Company, Belmont.

[3] Green, P.E. and Carroll, J.D. (1976) Mathematical Tools for Applied Multivariate Analysis. Academic Press, New York.

[4] El-Ghezawi, O.M.E. (1991) A Two-Stage Method for Eigenvalueigen Vector Assignment. Dirasat, 17, 65-77.

[5] D’azzo, J.J. and Houpis, C.H. (1995) Linear Control Systems: Analysis and Design. 4th Edition, McGraw-Hill, New York.

[6] El-Ghezawi, O.M.E. (2010) Unification and Improvement of Certain Methods for Eigenvalue Assignment. Dirasat, 37, 206-213.

[7] Ackermann, J. and Utkin, V.I. (1998) Sliding Mode Control Design Based on Ackermann’s Formula. IEEE Transactions on Automatic Control, 43, 234-237. http://dx.doi.org/10.1080/00207178708933729

[8] Petkov, P., Christov, N. and Konstantinov, M. (1991) Computational Methods for Linear Control Systems. Prentice Hall, Upper Saddle River.

[9] Lancaster, P. and Tismentasky, M. (1985) The Theory of matrices with Applications. 2nd Edition, Academic Press, New York.

[10] Schott, J.R. (1997) Matrix Analysis for Statistics. John Wiley, Hoboken.

[11] El-Ghezawi, O.M.E. (1997) Recursive and Modified Recursive Eigenstructure Assignment of Uncontrollable Systems. Dirasat, 24, 620-628.

[12] Liu, G.P. and Patton, R.J. (1998) Eigenstructure Assignment for Control System Design. John Wiley \& Sons, New York.

[13] White, B.A. (1995) Eigenstructure Assignment: A Survey. Proceedings of the Institution of Mechanical Engineers, 209, 1-11. http://dx.doi.org/10.1243/pime_proc_1995_209_357_02

[14] Sobel, K.M., Shapiro, E.Y. and Andry, A.N. (1994) Eigenstructure Assignment. International Journal of Control, 59, 13-37. http://dx.doi.org/10.1080/00207179408923068

[15] Friedland, B. (2005) Control System Design: Introduction to State Space Methods. Dover Publications, New York. 